\title{
A 42-year-old man with a rapidly growing lesion of the soft palate
}

\begin{abstract}
A 42-year-old previously well man was referred to our dermatology clinic for evaluation of a lesion on his soft palate. The lesion had appeared suddenly without precipitating trauma, had grown rapidly over 2 months and tended to bleed easily.

Examination revealed an erythematous, pedunculated lesion that measured $0.8 \mathrm{~mm}$ in diameter and was soft and mobile (Fig. 1). The lesion was excised using electrocautery. Histopathologic examination revealed a characteristic lobular proliferation of microvascular vessels forming tiny vascular lumina, ulceration with superficial neutrophilic infiltrates, marked stromal edema and irregular dilatation of blood vessels. These findings confirmed the clinical diagnosis of lobular capillary hemangioma (LCH). The patient had not experienced recurrence of the lesion 12 months after excision.
\end{abstract}

The term "lobular capillary hemangioma" is used synonymously with the more historically common term "pyogenic granuloma," which is based on the misunderstanding that the lesion represents a nonspecific granulation-tissue response to infection by pyogenic agents.

In fact, LCH is a common reactive angiomatous proliferation that occurs in the skin and mucous membranes. It may occur spontaneously but usually results from traumatic insult to the tissue. The most common sites are areas with an abundant blood supply that are vulnerable to trauma (e.g., the gingiva in denture wearers, fingers, lips, face and tongue).

LCH lesions are most common in children and young adults and in pregnant women. "Granuloma gravidarum" and "epulis gravidarum" have been

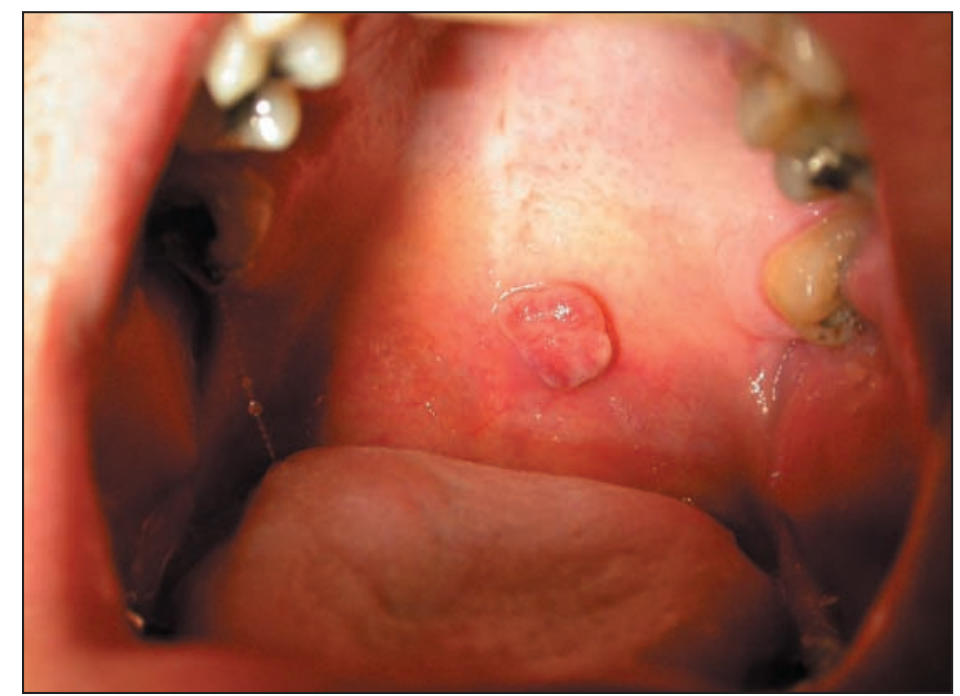

used to describe the lobular capillary hemangiomas on the gingiva that develop in up to $2.5 \%$ of pregnant women, often during the second and third trimester.

LCH initially appears as a red or reddish-brown sessile papule that is painless and pliable to the touch. The lesion grows quickly over a few weeks, becoming dome shaped and measuring 5$10 \mathrm{~mm}$ in diameter and sometimes evolving into a pedunculated nodule or papule $2-3 \mathrm{~cm}$ in diameter. Growth usually stabilizes after 3-4 weeks. The lesion may have a thick epidermis that surrounds the base like a collar. Bleeding is typical with minimal trauma. Usually a solitary lesion forms, but there are isolated cases of multiple lesions.

Although the exact cause is unknown, it has been hypothesized that localized trauma stimulates the release of angiogenic factors to induce LCH growth. Estrogen and progesterone have also been shown to enhance angiogenesis in traumatized mucous membranes, ${ }^{2}$ which may account for the increased frequency of these tumours in pregnant women.

The differential diagnosis of $\mathrm{LCH}$ includes cutaneous and mucosal cancers such as achromic melanoma, basal and squa- mous cell carcinoma, Kaposi's sarcoma and glomus tumours, and benign lesions such as hemangiomas and bacillary angiomatosis. LCH is distinguished clinically by its rapid appearance and growth over 1-2 months and for its tendency to bleed easily and copiously. Histopathologic examination is required for a correct diagnosis.

Prevention of LCH depends on eliminating sources of chronic trauma. Surgical excision, cryotherapy, pulsed dye laser therapy and carbon dioxide laser therapy are excellent treatment options with similar outcomes. Treatment may be challenging: the lesions tend to recur after excision in $10 \%-15 \%$ of cases.

\section{Vincenzo de Giorgi \\ Serena Sestini \\ Paolo Nardini \\ Paolo Carli \\ Department of Dermatology \\ University of Florence \\ Florence, Italy}

\section{References}

1. Northington ME, Huang CC. Pyogenic granuloma caused by exposed sternotomy wires. Dermatol Surg 2004;30:1572-3

2. Whitaker SB, Bouquot JE, Alimario AE, Whitaker TJ Jr. Identification and semiquantification of estrogen and progesterone receptors in pyogenic granulomas of pregnancy. Oral Surg Oral Med Oral Pathol 1994;78:755-60. 